\title{
Implications of Flavor Dynamics for Fermion Triplet Leptogenesis
}

\author{
D. Aristizabal Sierra ${ }^{a, b}$, , Jernej F. Kamenik ${ }^{2}{ }^{2}$ and Miha Nemevšek ${ }^{d, c}$ 多 \\ ${ }^{a}$ Universite de Liege, Institut de physique Bat B5, Sart Tilman B-4000 Liege 1, Belgium. \\ ${ }^{b}$ INFN, Laboratori Nazionali di Frascati,C.P. 13, I00044 Frascati, Italy. \\ ${ }^{c} J$. Stefan Institute, 1000 Ljubljana, Slovenia. \\ ${ }^{d}$ II. Institut für Theoretische Physik, Universität Hamburg, Luruper Chaussee 149, \\ 22761 Hamburg, Germany.
}

\begin{abstract}
We analyze the importance of flavor effects in models in which leptogenesis proceeds via the decay of Majorana electroweak triplets. We find that depending on the relative strengths of gauge and Yukawa reactions the $B-L$ asymmetry can be sizably enhanced, exceeding in some cases an order of magnitude level. We also discuss the impact that such effects can have for $\mathrm{TeV}$-scale triplets showing that as long as the $B-L$ asymmetry is produced by the dynamics of the lightest such triplet they are negligible, but open the possibility for scenarios in which the asymmetry is generated above the $\mathrm{TeV}$ scale by heavier states, possibly surviving the $\mathrm{TeV}$ triplet related washouts. We investigate these cases and discuss how they can be disentangled by using Majorana triplet collider observables and, in the case of minimal type III see-saw models even through lepton flavor violation observables.
\end{abstract}

\footnotetext{
${ }^{1}$ e-mail address: Diego.Aristizabal@lnf.infn.it

${ }^{2}$ e-mail address: jernej.kamenik@ijs.si

${ }^{3}$ e-mail address: miha.nemevsek@ijs.si
} 


\section{Introduction}

Measurements from light element abundances and the cosmic microwave background radiation allow to determine the cosmic baryon asymmetry, $Y_{\Delta_{B}}=(8.75 \pm 0.23) \times 10^{-11}[1$. The conditions under which this asymmetry can be dynamically generated (baryogenesis) are well known [2] and depending on how they are realized different mechanisms for baryogenesis can be defined [3].

Leptogenesis is a mechanism in which an initial lepton asymmetry is partially reprocessed into a baryon asymmetry by nonperturbative sphaleron interactions [4]. Qualitatively a net non-zero lepton asymmetry can be generated in any framework containing interactions that: $(i)$ break lepton number; $(i i)$ violate $\mathrm{CP}$; $($ iii $)$ depart from thermal equilibrium at some stage in the cosmic evolution. In principle, these conditions are satisfied in any neutrino mass model in which light neutrinos acquire Majorana masses and therefore in leptogenesis two unrelated puzzles, the origin of the Cosmic baryon asymmetry and of neutrino masses, are linked together. In the standard approach, in which light neutrinos acquire Majorana masses via the type I see-saw model [5], leptogenesis takes place via the CP violating out-of-equilibrium decays of heavy standard model fermionic singlets [6]. This scenario for leptogenesis has been widely discussed in the literature [7] and indeed a lot of progress in the understanding of the generation of the lepton asymmetry within this scenario has been achieved [8, 9, 10, 11, 12, 13, 14, 15].

Given that qualitatively the conditions for producing a lepton asymmetry are also fulfilled in other see-saw realizations (type II [16] and type III [17]) one is tempted to extend the standard type I analysis to these cases, and in fact studies of such scenarios have been considered [18, 19, 20, 21, 22, 23]. In these models the lepton asymmetry is generated via the dynamics of either a scalar (type II) or a Majorana fermion (type III) $S U(2)$ triplet and thus the major difference between these cases and the standard one arises from the fact that both, the scalar and fermion, couple to standard model (SM) gauge bosons. At high temperatures gauge reactions are much faster than the expansion rate of the Universe implying that no asymmetry can be generated at this stage. As the temperature drops, thermalization of the triplet distribution becomes less efficient and depending on the strength of the Yukawa interactions the generation of the lepton asymmetry can proceed either after the decoupling of gauge reactions or after the Yukawa interactions freeze-out [21].

In this paper we focus on leptogenesis in type III see-saw (fermionic triplet leptogenesis). In particular, we analyze the dynamics of lepton flavor in the generation of the lepton asymmetry and the implications that such effects could have for TeV-scale triplets. As in the standard case when the lightest triplet mass $\left(M_{T_{1}}\right)$ is below $10^{12} \mathrm{GeV}$ the charged lepton Yukawa interactions that are sufficiently fast project the lepton and anti-lepton quantum states produced in $T_{1}$ decays into their flavor components before they can re-scatter [8, 10, 11]. In the standard case the fermionic singlet dynamics is completely determined by Yukawa reactions and accordingly the impact of flavor effects becomes well pronounced. In contrast, in the fermionic triplet leptogenesis case, since the dynamics of the triplet is not completely determined by its Yukawa interactions, it is not entirely clear whether light flavor effects could yield a sizable enhancement of the final 
lepton asymmetry regardless of the strength of the Yukawa reactions. Here we will show that the inclusion of flavor may in general produce an enhancement of the asymmetry, but that relevant effects are possible only when the strength of the triplet Yukawa interactions are such that they are still active when gauge reactions decouple.

In the case of a quasi-degenerate fermionic singlet mass spectrum the resonant enhancement of the $\mathrm{CP}$ violating asymmetry allows for successful leptogenesis even when the fermionic singlet masses are well below $1 \mathrm{TeV}$ [24, 25]. In fermionic triplet leptogenesis on the other hand, the efficiency due to gauge reactions strongly depends on $M_{T_{1}}$ and it is drastically diminished when $\mathcal{O}\left(M_{T_{1}}\right) \sim \mathrm{TeV}$. In the one flavor approximation in ref. [23] it was pointed out that this constraint in conjunction with sphaleron decoupling implies that the correct amount of baryon asymmetry can only be generated for $M_{T_{1}} \gtrsim$ 1.6 TeV. As we will show, as long as leptogenesis proceeds through $T_{1}$ dynamics, this bound remains valid even when flavor effects are accounted for. However, this does not necessarily imply that the observation of fermionic triplets at the LHC would exclude the possibility of successful leptogenesis. Once flavor effects are taken into account the possibility of high scale leptogenesis remain plausible. As will be discussed, in that case, the $B-L$ asymmetry produced above the $\mathrm{TeV}$-scale (e.g. in $T_{2}$ decays) can survive $T_{1}$ related washouts only for particular flavor structures. These structures constrain the dynamics of $T_{1}$ leading to experimental signatures which could be used to constrain leptogenesis models.

The rest of this paper is organized as follows. In section 2 we discuss the generalities of type III see-saw and review the generation of the $B-L$ asymmetry in the one flavor approximation, we also determine the regions where Yukawa reactions remain active after gauge interactions decouple. In section 3 we study the generation of the flavored $B-L_{i}$ asymmetries showing how the inclusion of light flavors in the analysis may impact the final asymmetry. Section 3.1 is devoted to the analysis of high scale $\left(T_{2}\right)$ leptogenesis, section 3.2 to the possible collider patterns of $T_{1}$, induced by requiring that the asymmetry generated at a higher scale survive $T_{1}$ related washouts, and in section 3.3 we discuss the interplay between conditions for successful high scale leptogenesis and lepton flavor violating observables present in minimal type III see-saw models. In section 4 we summarize and present our conclusions. In appendix $\mathrm{A}$ we present our conventions and definitions used in our numerical calculations.

\section{Generalities}

From a bottom-up approach the type III see-saw model is a simple extension of the SM that contains $N_{T}$ additional fermionic $S U(2)$ triplets $\left(T_{\alpha}\right)$ with vanishing hypercharge ${ }^{1}$. In the basis in which the Majorana mass matrix for the triplets $M_{T_{\alpha}}$ is real and diagonal the interactions induced by the new states are given by the following Lagrangian

$$
\mathcal{L}_{T}=i \operatorname{Tr} \bar{T}_{\alpha} \not D T_{\alpha}-\lambda_{i \alpha}^{*} \bar{\ell}_{i} T_{\alpha} \widetilde{H}-\frac{1}{4} \operatorname{Tr} T_{\alpha}^{\dagger} C M_{T_{\alpha}} T_{\alpha}+\text { h.c. },
$$

\footnotetext{
${ }^{1}$ Consistency with neutrino data [26] requires at least two triplets. However, apart from this constraint, the number of fermion triplets is arbitrary.
} 
where $\ell=(\nu, l)^{T}$ and $H=\left(h^{+}, h^{0}\right)^{T}$ are the lepton and Higgs $S U(2)$ doublets $(\widetilde{H}=$ $\left.i \tau_{2} H^{*}\right)$. The triplets can be written as a matrix

$$
T_{\alpha}=\tau^{A} T_{\alpha}^{A}=\left(\begin{array}{cc}
T_{\alpha}^{0} & \sqrt{2} T_{\alpha}^{+} \\
\sqrt{2} T_{\alpha}^{-} & -T_{\alpha}^{0}
\end{array}\right)
$$

where $T^{0}=T^{3}, T^{ \pm}=\left(T^{1} \mp i T^{2}\right) / \sqrt{2}$ and $C$ is the charge conjugation operator. In this notation, the covariant derivative is defined as $D_{\mu}=\partial_{\mu}-i g \tau^{A} W_{\mu}^{A} / 2$. The Greek indices $\alpha, \beta \ldots=1, \ldots, N_{T}$ are used to label the different fermionic triplet generations, Latin indices $i, j, \ldots$ for the lepton flavors $e, \mu, \tau$.

The Majorana triplets' mass terms break lepton number and thus, after electroweak symmetry breaking, induce Majorana neutrino masses for the left-handed neutrinos. In the basis $\left(\nu_{L i}, T_{\alpha}^{0}\right)$, where the heavy neutrino mass matrix is diagonal, the neutral $(3+$ $\left.N_{T}\right) \times\left(3+N_{T}\right)$ fermion mass matrix reads

$$
M_{\boldsymbol{N}}=\left(\begin{array}{cc}
0 & v \lambda \\
v \lambda^{T} & \hat{M}_{\boldsymbol{T}}
\end{array}\right)
$$

where $v$ is the SM Higgs vacuum expectation value, $v \simeq 174 \mathrm{GeV}$. Accordingly the effective light neutrino mass matrix is given by

$$
\boldsymbol{m}_{\boldsymbol{\nu}}^{\mathrm{e} f f}=-v^{2} \boldsymbol{\lambda} \cdot \hat{M}_{\boldsymbol{T}}^{-1} \cdot \boldsymbol{\lambda}^{T}
$$

In general the Yukawa coupling matrix $\boldsymbol{\lambda}$ is a complex $3 \times N_{T}$ matrix in flavor space and therefore contains new sources of CP violation, as can be clearly seen by expressing $\lambda$ by means of the Casas-Ibarra parametrization [27]:

$$
\lambda=U^{*} \cdot \sqrt{\hat{m}_{\nu}} \cdot R \cdot \sqrt{\hat{M}_{T}}
$$

which ensures that the correct low energy parameters are obtained. Here $\boldsymbol{U}$ is the leptonic mixing matrix which diagonalizes the effective neutrino mass matrix $\boldsymbol{m}_{\boldsymbol{\nu}}{ }^{\text {eff }}$ and is fixed by low energy observables (three light neutrino mixing angles, one Dirac and one or two Majorana CP violating phases). The matrix $R$ is an orthogonal complex matrix defined by $N_{T}\left(N_{T}-1\right) / 2$ complex parameters.

The CP violating sources contained in $\lambda$ induce two-body $\mathrm{CP}$ violating decays of $T_{\alpha}$. The tree level decay width for these processes reads

$$
\Gamma_{\alpha}=\sum_{j} \Gamma\left(T_{\alpha} \rightarrow \bar{\ell}_{j} \widetilde{H}, \ell_{j} \widetilde{H}^{\dagger}\right)=\frac{M_{T_{\alpha}}}{8 \pi} \sum_{i=e, \mu, \tau} \lambda_{i \alpha}^{*} \lambda_{i \alpha}=\frac{M_{T_{\alpha}}^{2}}{8 \pi v^{2}} \sum_{i=e, \mu, \tau} \widetilde{m}_{i \alpha}
$$

where

$$
\tilde{m}_{i \alpha}=\frac{v^{2}}{M_{T_{\alpha}}} \lambda_{i \alpha}^{*} \lambda_{i \alpha} \text { and } \quad \widetilde{m}_{\alpha}=\sum_{i=e, \mu, \tau} \tilde{m}_{i \alpha}
$$

In terms of (5) these parameters are determined by low energy observables and by the entries of the matrix $\boldsymbol{R}$. Given that such entries can be arbitrarily large, without affecting 

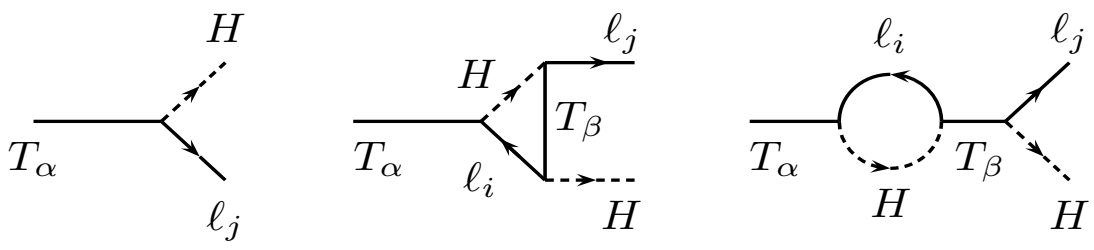

Figure 1: Diagrams generating the flavored CP violating asymmetries $\epsilon_{T_{\alpha}}^{\ell_{i}}$.

the light neutrino masses, light triplets $(\mathcal{O}(\mathrm{TeV}))$ not necessarily imply small values of $\widetilde{m}_{\alpha}$.

The CP violating asymmetries for $T_{\alpha}$ decays into $\ell_{j}$ lepton flavor arise, as in type I see-saw [28], through the interference between the tree level and the one-loop vertex (V) and wave-function (W) diagrams (see fig. 1). The corresponding expressions are given by

$$
\begin{aligned}
\epsilon_{T_{\alpha}}^{\ell_{j}(\mathrm{~V})} & =\frac{1}{8 \pi} \sum_{\beta \neq \alpha} \frac{\operatorname{Im}\left[\sqrt{\omega_{\beta}}\left(\lambda^{\dagger} \lambda\right)_{\beta \alpha} \lambda_{j \beta}^{*} \lambda_{j \alpha}\right]}{\left(\lambda^{\dagger} \lambda\right)_{\alpha \alpha}} f\left(\omega_{\beta}\right), \\
\epsilon_{T_{\alpha}}^{\ell_{j}(\mathrm{~W})} & =-\frac{1}{8 \pi} \sum_{\beta \neq \alpha} \frac{\operatorname{Im}\left\{\left[\left(\lambda^{\dagger} \lambda\right)_{\alpha \beta}+\sqrt{\omega_{\beta}}\left(\lambda^{\dagger} \lambda\right)_{\beta \alpha}\right] \lambda_{j \beta}^{*} \lambda_{j \alpha}\right\}}{\left(\lambda^{\dagger} \lambda\right)_{\alpha \alpha}} g\left(\omega_{\beta}\right),
\end{aligned}
$$

where $\omega_{\beta}=M_{T_{\beta}}^{2} / M_{T_{\alpha}}^{2}$ and the loop functions are given by

$$
\begin{aligned}
& f\left(\omega_{\beta}\right)=\left(1+\omega_{\beta}\right) \ln \left(\frac{\omega_{\beta}+1}{\omega_{\beta}}\right)-1, \\
& g\left(\omega_{\beta}\right)=\frac{\omega_{\beta}-1}{\left(\omega_{\beta}-1\right)^{2}+a_{\Gamma_{\beta}}^{2}}
\end{aligned}
$$

with $a_{\Gamma_{\beta}}=\Gamma_{\beta} / M_{\alpha}$. In the case of a quasi-degenerate triplet spectrum $\left(\sqrt{\omega_{\beta}} \sim 1+a_{\Gamma_{\beta}}\right)$ the wave-function piece is resonantly enhanced. In such cases, depending on the size of the CP violating phases, the flavored CP asymmetries, entirely determined by the wavefunction piece, can be $\mathcal{O}(1)$ (see ref. [24, 25] for a thorough discussion of such possibility within type I see-saw). In the case of a hierarchical triplet spectrum $\left(\omega_{\beta} \gg 1\right.$ with $\left.\beta>\alpha\right)$ $a_{\Gamma_{\beta}}$ can be neglected and the loop functions expanded in powers of $\omega_{\beta}^{-1}$. At leading order the flavored CP violating asymmetries can be expressed as

$$
\epsilon_{T_{\alpha}}^{\ell_{j}}=-\frac{1}{8 \pi\left(\lambda^{\dagger} \lambda\right)_{\alpha \alpha}} \sum_{\beta \neq \alpha} \operatorname{Im}\left\{\left[\frac{\left(\lambda^{\dagger} \lambda\right)_{\alpha \beta}}{\omega_{\beta}}+\frac{\left(\lambda^{\dagger} \lambda\right)_{\beta \alpha}}{2 \sqrt{\omega_{\beta}}}\right] \lambda_{j \beta}^{*} \lambda_{j \alpha}\right\} .
$$

Some comments are in order regarding the different terms in this expression. Since the term $\sum_{\beta}\left(\lambda^{\dagger} \lambda\right)_{\alpha \beta}\left(\lambda^{\dagger} \lambda\right)_{\beta \alpha}$ is real, the first term only contributes to the flavored CP violating asymmetries [11, 28]. Although, apart from the kinetic term, the Lagrangian in eq. (1) has the same structure as the Lagrangian in type I see-saw, the contractions of the $S U(2)$ indices in the Yukawa interaction terms are different and consequently the second term in 10$)$ is a factor 3 smaller than the corresponding one in the standard case ${ }^{2}$.

\footnotetext{
${ }^{2}$ In contrast to the type I see-saw case, due to these contractions, there is a relative sign between the wave-function and vertex pieces. Thus, at leading order, $\epsilon_{T_{\alpha}}^{\ell_{j}(\mathrm{~W})} \rightarrow 1 / \sqrt{\omega_{\beta}}$ whereas $\epsilon_{T_{\alpha}}^{\ell_{j}(\mathrm{~V})} \rightarrow-1 / 2 \sqrt{\omega_{\beta}}$
} 
In the strongly hierarchical limit, where the first term in 10 can be neglected, and assuming a normal hierarchical light neutrino spectrum it can be shown that as in the standard case there is an upper bound on the flavored CP violating asymmetries, namely [10]

$$
\epsilon_{T_{\alpha}}^{\ell_{j}} \lesssim 10^{-5}\left(\frac{M_{T_{\alpha}}}{10^{10} \mathrm{GeV}}\right)\left(\frac{m_{3}}{1 \mathrm{eV}}\right) \frac{\widetilde{m}_{j \alpha}}{\widetilde{m}_{\alpha}} .
$$

Finally, the total CP violating asymmetry can be obtained from 10 by summing over the flavor index $j$ [21]

$$
\epsilon_{T_{\alpha}}=-\frac{1}{16 \pi} \sum_{\beta} \frac{1}{\sqrt{\omega_{\beta}}} \frac{\operatorname{Im}\left[\left(\lambda^{\dagger} \lambda\right)_{\beta \alpha}^{2}\right]}{\left(\lambda^{\dagger} \lambda\right)_{\alpha \alpha}} .
$$

The major quantitative difference between the standard leptogenesis scenario and fermion triplet leptogenesis lies in the fact that fermion triplets couple to SM gauge bosons. At $z=M_{T} / T \ll 1$ gauge reactions thermalize the triplet distribution meaning that no asymmetry is produced at high $T$. Gauge reactions decouple at temperatures when

$$
\frac{\Gamma_{A}}{H}=\frac{\gamma_{A}}{n_{T}^{\mathrm{Eq}} H} \lesssim 1,
$$

where $\gamma_{A}$ is the gauge reaction density, $H$ is the expansion rate of the Universe and $n_{T}^{\mathrm{Eq}}$ is the equilibrium triplet number density (see appendix A). Thus, if at this stage inverse decay processes $\ell H \rightarrow T$ are decoupled as well $\left(\gamma_{D} / n_{\ell}^{\mathrm{Eq}} H \lesssim 1\right.$, where $\gamma_{D}$ is the decay reaction density), the $\mathrm{CP}$ violating out-of-equilibrium decays of the triplets will produce a $B-L$ asymmetry. Conversely, if inverse decays are still active when gauge reactions decouple the $B-L$ asymmetry will be generated at lower temperatures, after inverse decays switch off. Since $\gamma_{A} / \gamma_{D} \sim g^{4} / M_{T} \widetilde{m}$, as $M_{T}$ decreases only large values of $\widetilde{m}$ are able to mantain inverse decays active after gauge reaction decoupling takes place. Fig. 2 shows an illustrative example where it can be seen that for $M_{T}=10^{12} \mathrm{GeV}$, inverse decay processes are still active after gauge interaction decoupling provided $\widetilde{m} \sim 5 \times 10^{-3}$ $\mathrm{eV}$, whereas for $M_{T}=10^{10} \mathrm{GeV}$ this occurs for a $\widetilde{m}$ an order of magnitude larger. Independently of how leptogenesis proceeds, a precise determination of the generated $B-L$ asymmetry requires a numerical treatment of the corresponding Boltzmann equations, which we now discuss in turn.

\subsection{Generation of the $B-L$ asymmetry}

Although in this section we will focus on the case in which the asymmetry is generated along one specific flavor direction, we will write flavor dependent Boltzmann equations as they will be used in sec. 3. Considering only triplet annihilations (mediated by SM gauge bosons), decays (induced by the Yukawa couplings $\lambda$ ) and $\Delta L=2$ scatterings $\left(\ell_{j} \widetilde{H}^{\dagger} \leftrightarrow \ell_{i} \widetilde{H}^{\dagger} \text { and } \bar{\ell}_{j} \widetilde{H} \leftrightarrow \bar{\ell}_{i} \widetilde{H}\right)^{3}$ the network of flavor dependent Boltzmann equations

\footnotetext{
${ }^{3}$ Although we work at $\mathcal{O}\left(\lambda^{2}\right)$ the inclusion of these processes is mandatory to obtain Boltzmann equations with the correct thermodynamical behavior [29].
} 


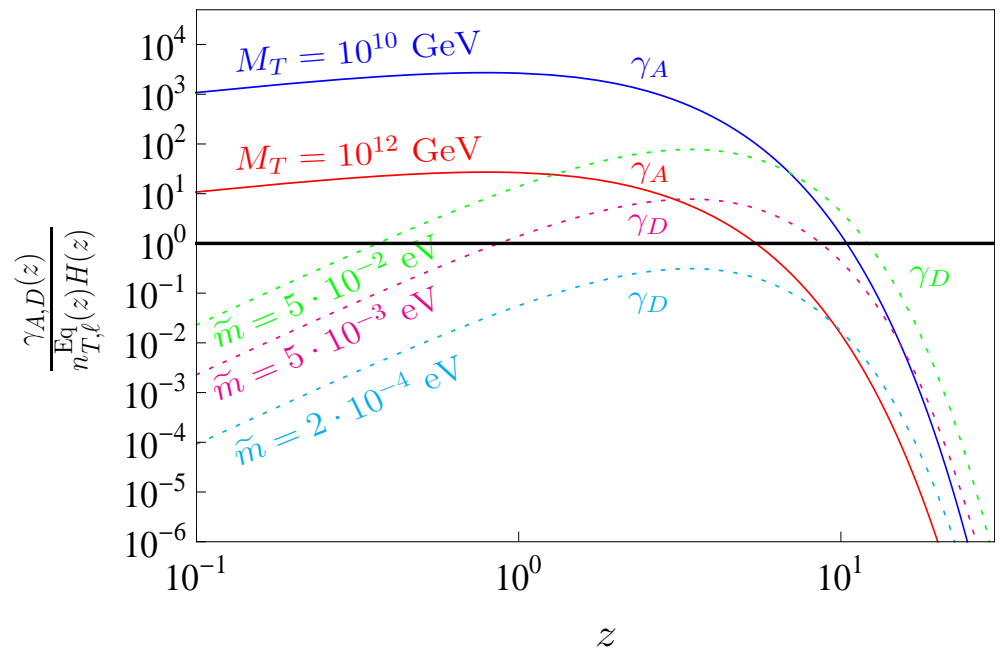

Figure 2: Evolution of gauge and Yukawa reaction densities $\left(\gamma_{A}, \gamma_{D}\right)$ with $z$. The horizontal black line indicates when do either gauge or Yukawa reactions go out of equilibrium.

at $\mathcal{O}\left(\lambda^{2}\right)$, assuming a vanishing Higgs number asymmetry [12], can be written as

$$
\begin{aligned}
& \frac{d Y_{T_{\alpha}}}{d z_{\alpha}}=-\frac{1}{s H z_{\alpha}}\left[\left(\frac{Y_{T_{\alpha}}}{Y_{T_{\alpha}}^{\mathrm{Eq}}}-1\right) \gamma_{D_{\alpha}}+\left(\frac{Y_{T_{\alpha}}^{2}}{\left(Y_{T_{\alpha}}^{\mathrm{Eq}}\right)^{2}}-1\right) \gamma_{A_{\alpha}}\right], \\
& \frac{d Y_{\Delta_{i}}}{d z_{\alpha}}=-\frac{1}{s H z_{\alpha}}\left[\left(\frac{Y_{T_{\alpha}}}{Y_{T_{\alpha}}^{\mathrm{Eq}}}-1\right) \epsilon_{T_{\alpha}}^{\ell_{i}}+\frac{K_{i \alpha}}{2 Y_{\ell}^{\mathrm{Eq}}} \sum_{j=e, \mu, \tau} C_{i j}^{\ell} Y_{\Delta_{j}}\right] \gamma_{D_{\alpha}} .
\end{aligned}
$$

Here $z_{\alpha}=M_{T_{\alpha}} / T, Y_{X}=n_{X} / s$ and $Y_{\Delta_{i}}=Y_{\Delta_{B / 3-L_{i}}}$ with $Y_{L_{i}}=2 Y_{\ell_{i}}+Y_{e_{i}}$ (the lepton asymmetry distributed in left and right handed degrees of freedom). The flavor projectors $K_{i \alpha}$ are defined as follows [12]:

$$
K_{i \alpha}=\frac{\lambda_{i \alpha}^{*} \lambda_{i \alpha}}{\left(\lambda^{\dagger} \lambda\right)_{\alpha \alpha}}=\frac{\widetilde{m}_{i \alpha}}{\widetilde{m}_{\alpha}},
$$

note that $\sum_{i=e, \mu, \tau} K_{i \alpha}=1$. The numerical coefficients $C_{i j}^{\ell}$, that relate $Y_{\ell_{i}}$ with $Y_{\Delta_{i}}$ according to [11]

$$
Y_{\ell_{i}}=-\sum_{j} C_{i j}^{\ell} Y_{\Delta_{j}}
$$

couple the different differential equations in (15) and are determined by the reactions that at a certain temperature regime are in equilibrium [8, 11, 10], finally

$$
\gamma_{D_{\alpha}}=\sum_{i=e, \mu, \tau} \gamma_{D_{i \alpha}} .
$$

By integrating eq. (15) the resulting $Y_{\Delta_{B-L}}$ asymmetry can be expressed according to (see appendix A)

$$
Y_{\Delta_{B-L}}=\sum_{i=e, \mu, \tau} Y_{\Delta_{i}}=3 \times \sum_{i=e, \mu, \tau} \epsilon_{T_{\alpha}}^{\ell_{i}} Y_{T_{\alpha}}^{\mathrm{Eq}} \eta_{i \alpha},
$$



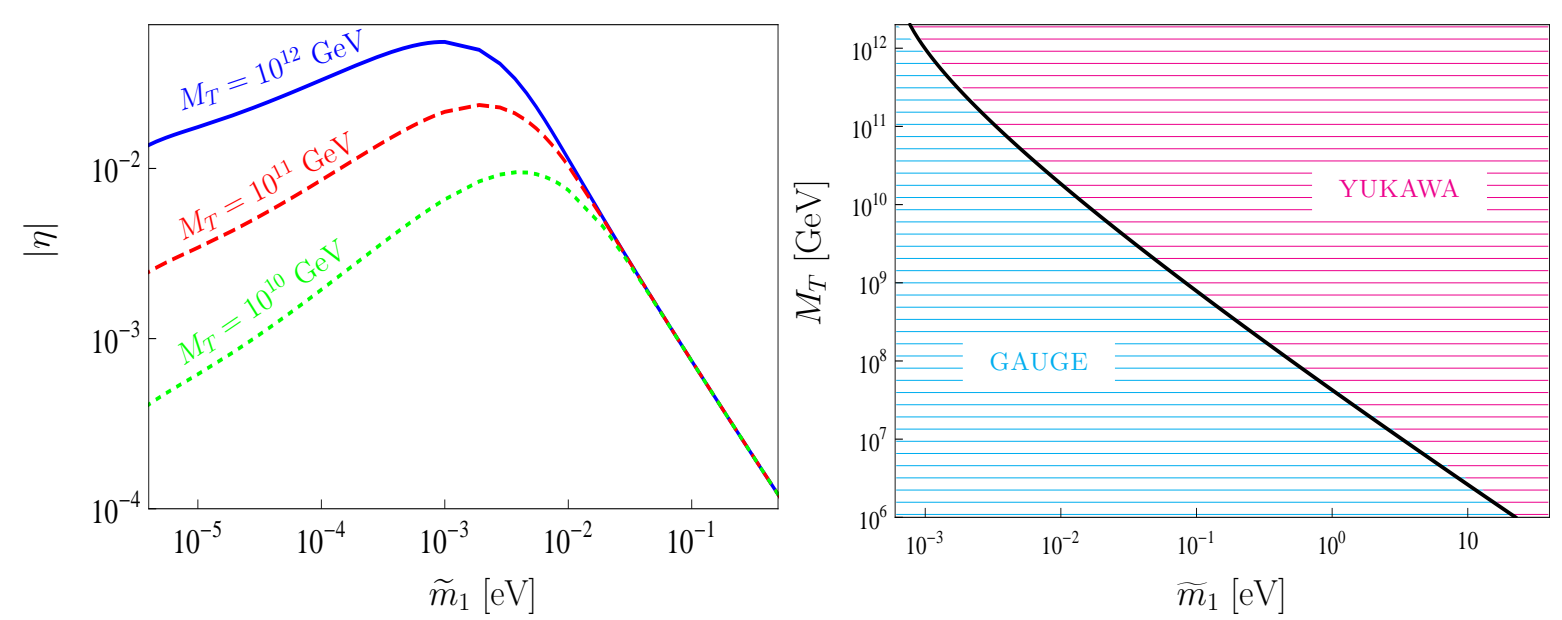

Figure 3: Efficiency factor as a function of $\widetilde{m}_{1}$ in the flavor aligned case (left panel) and regions for which gauge interactions freeze out after (lower region) and before (upper region) Yukawa reaction decoupling (right panel).

where the factor 3 accounts for the three $S U(2)$ degrees of freedom of the triplets and $\eta_{i \alpha}$ denotes the efficiency factor that accounts for the $T_{\alpha}$ generated asymmetry in flavor $i$ that survives washouts. In the case of a hierarchical spectrum $\left(M_{T_{1}} \ll M_{T_{\alpha>1}}\right)$, neglecting the dynamics of the heavier triplets, leptogenesis proceeds entirely through $T_{1}$ dynamics. Compared with the standard case the amount of $Y_{\Delta_{B-L}}$ is smaller and its value can strongly depend on $M_{T_{1}}$ (depending on the size of $\widetilde{m}_{1}$ ). In order to demonstrate this we have numerically solved eqs. (14) and (15) assuming alignment along flavor $j\left(K_{j 1}=\delta_{j 1}\right)$ and with $C_{i j}^{\ell}=\delta_{i j}$. Figure 3 (left panel) shows the evolution of the efficiency factor $(\eta)$ with $\widetilde{m}_{1}$ for several values of $M_{T_{1}}$. It can be seen that the dependence on $M_{T_{1}}$ is stronger for small values of $\widetilde{m}_{1}$ and less pronounced as $\widetilde{m}_{1}$ increases, being completely independent of $M_{T_{1}}$ for large values of $\widetilde{m}_{1}$, which means there is a $\widetilde{m}_{1}^{\min }$ above which leptogenesis proceeds as in the standard case ${ }^{4}$. This implies that although gauge processes introduce a dependence on the triplet mass this dependence disappears as soon as the gauge interactions are subdominant with respect to Yukawa reactions. Thus, in the fermionic triplet leptogenesis the generation of the $B-L$ asymmetry can proceed either in a region determined by the condition $\widetilde{m}_{1}<\widetilde{m}_{1}^{\text {min }}$ or conversely in a region defined by $\widetilde{m}_{1}>\widetilde{m}_{1}^{\text {min }}$. The determination of $\widetilde{m}_{1}^{\mathrm{min}}$, for a given triplet mass, can be done as follows. From the gauge decoupling condition $\gamma_{A} / n_{T}^{\mathrm{Eq}} H \lesssim 1$ the corresponding $z=z_{A \text {-dec }}$ at which gauge reactions go out of equilibrium can be calculated. The value $\widetilde{m}_{1}^{\min }$ can be computed by requiring that the Yukawa interactions are still active at $z_{A-\text { dec }}$ i.e $\left.\left(\gamma_{D} / n_{\ell}^{\mathrm{Eq}} H\right)\right|_{z=z_{A-\mathrm{dec}}} \gtrsim 1$. Figure 3 shows the values $\left(\widetilde{m}_{1}, M_{T_{1}}\right)$ for which the $B-L$ asymmetry either depends on $M_{T_{1}}$ ("gauge region") or is entirely determined by $\widetilde{m}_{1}$ ("Yukawa region").

\footnotetext{
${ }^{4}$ In standard leptogenesis at $\mathcal{O}\left(\lambda^{2}\right)$ the efficiency does not depend on the fermionic singlet mass at all.
} 


\section{Including Flavor}

Flavor effects become relevant at temperatures below $10^{13} \mathrm{GeV}$ when bottom and tau Yukawa interactions enter into thermodynamic equilibrium [8, 10, 11]. For definiteness, from now on, we will focus in the temperature window $10^{9} \mathrm{GeV} \lesssim M_{T_{1}} \lesssim 10^{12} \mathrm{GeV}$ at which, in addition to the bottom and tau Yukawa processes, also electroweak sphalerons are already in thermal equilibrium. In this regime the $B-L$ asymmetry is distributed along $\ell_{\tau}$ and $\ell_{1}$ (an admixture of muon and electron flavors). The determination of the total asymmetry in this case is therefore a two flavor problem $\left(\ell_{\tau}, \ell_{1}\right)$ and the network of Boltzmann equations in (14)-(15) consist of three coupled differential equations in the variables $Y_{T_{1}}, Y_{\Delta_{\tau}}$ and $Y_{\Delta_{1}}$ where the flavored asymmetries are coupled by the flavor coupling matrix [11]

$$
C^{\ell}=\frac{1}{460}\left(\begin{array}{cc}
196 & -24 \\
-9 & 156
\end{array}\right)
$$

obtained from the chemical equilibrium conditions imposed by reactions that in the corresponding temperature window are faster than the expansion rate [30]. According to the discussion of the previous section, in triplet fermion leptogenesis the asymmetry can proceed in one of the two following regimes: (a) Gauge reaction decoupling takes place after Yukawa induced inverse decays have gone out of equilibrium - "gauge region"; $(b)$ Gauge reactions freeze-out when inverse decay processes are still active - "Yukawa region". In scenario $(a)$ since the the triplet abundance is efficiently diminished by gauge boson mediated annihilations the effects of flavor are expected to be small, or even negligible. Instead, in scenario $(b)$ since at $z_{1} \gg 1$ the dynamics of $T_{1}$ is entirely determined by Yukawa reactions, flavor effects may be sizable and can be prominent as long as

$$
\left|\epsilon_{T_{1}}^{\ell_{j}}\right|>\left|\epsilon_{T_{1}}^{\ell_{i}}\right| \quad \text { and } \quad K_{j 1} \ll K_{i 1} \text {. }
$$

In order to show this is in fact the case, we have calculated the evolution of the $Y_{\Delta_{B-L}}(z)$ asymmetry for $M_{T_{1}}=10^{12} \mathrm{GeV}$ and $\widetilde{m}_{1}=2 \times 10^{-4}, 5 \times 10^{-2} \mathrm{eV}$ by numerically integrating eqs. (14) and (15), and assuming the following flavor configuration $K_{11}=0.99,\left(K_{\tau 1}=\right.$ $\left.1-K_{11}\right)$ and $\epsilon_{T_{1}}^{\ell_{1}}=-0.1 \times \epsilon_{T_{1}}$ and $\epsilon_{T_{1}}^{\ell_{\tau}}=1.1 \times \epsilon_{T_{1}}$ with $\epsilon_{T_{1}}=10^{-5}$. The result is displayed in fig. 4 where, in addition to the resulting asymmetry in the flavored case, we also show the corresponding asymmetry in the fully aligned case to facilitate the comparison between both cases. As can be seen in fig. 4 (left panel) for $\widetilde{m}_{1}=2 \times 10^{-4} \mathrm{eV}$ (regime $(a)$ ) flavor effects are small, producing only a $\sim 5 \%$ enhancement. In contrast, for $\widetilde{m}_{1}=5 \times 10^{-2}$ $\mathrm{eV}$, and given that the flavor configuration we chose satisfies (21), flavor effects produce a two orders of magnitude enhancement of the final $B-L$ asymmetry: $Y_{\Delta_{B-L}}^{\mathrm{Alig}}=2.1 \times 10^{-10}$ whereas $Y_{\Delta_{B-L}}^{\text {Flav }}=3.1 \times 10^{-8}$. As in the standard case the effects of flavor could be even larger once the muon Yukawa coupling enters into thermodynamical equilibrium since in that case two flavor projectors can be simultaneously small.

As previously discussed, the minimum value of $\widetilde{m}_{1}$ for which flavor effects can produce a sizable enhancement of the $B-L$ asymmetry is not unique and depends on the triplet mass. Fig. 5 shows the behavior of the $B-L$ asymmetry with $\widetilde{m}_{1}$. As can be seen for $M_{T}=10^{12} \mathrm{GeV}$ flavor effects start playing a role at $\widetilde{m}_{1} \sim 10^{-3} \mathrm{eV}$ and produce a 

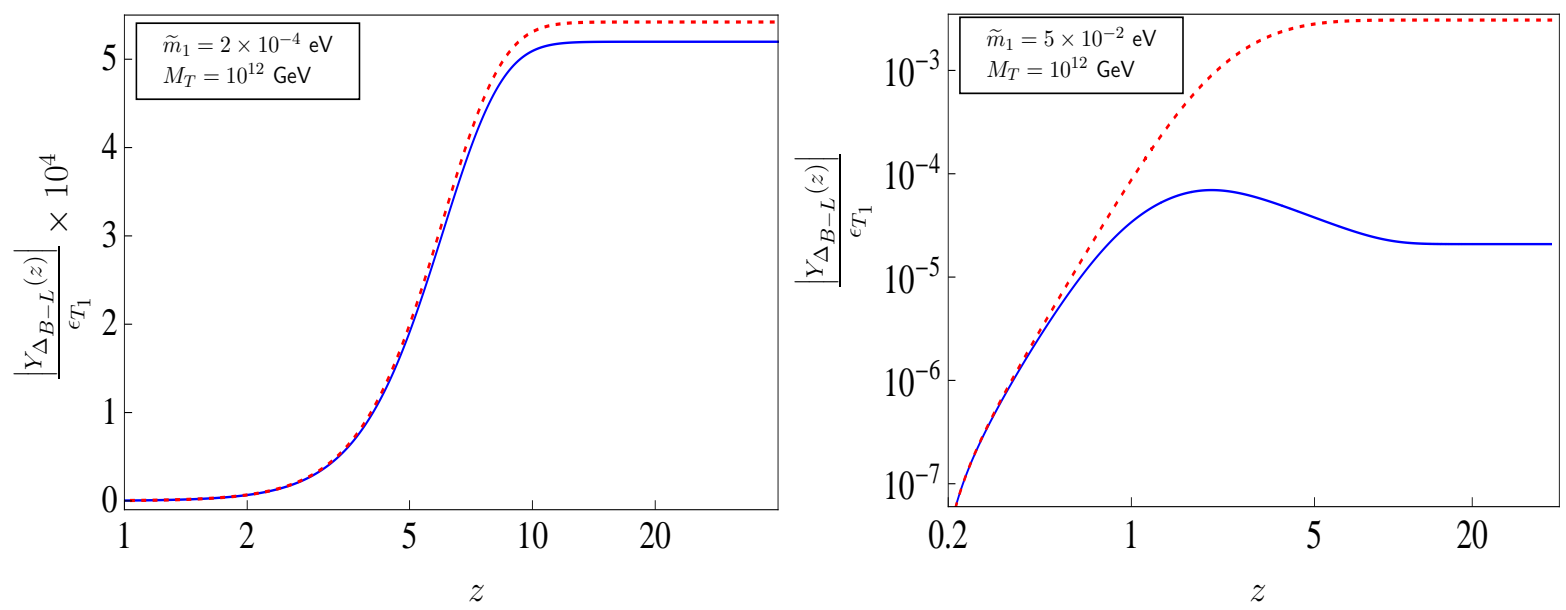

Figure 4: $Y_{\Delta_{B-L}}(z) / \epsilon_{T_{\alpha}}$ vs $z$ for aligned (solid blue line) and flavored (dotted red line) cases in the temperature regime $10^{10} \mathrm{GeV} \lesssim T \lesssim 10^{12} \mathrm{GeV}$. The flavored CP asymmetries are fixed as $\epsilon_{T_{1}}^{\ell_{1}}=-0.1 \times \epsilon_{T_{1}}, \epsilon_{T_{1}}^{\ell_{\tau}}=1.1 \times \epsilon_{T_{1}}$ and $\epsilon_{T_{1}}=10^{-5}$ whereas the flavor projectors are accordingly $K_{11}=0.99$ and $K_{1 \tau}=0.01$.

strong enhancement above this value i.e. well inside the "Yukawa region" (see fig. 3 right panel). For $M_{T}=10^{10} \mathrm{GeV}$, as anticipated, the related effects start being relevant for larger values of $\widetilde{m}_{1}$, again well within the "Yukawa region".

\subsection{Implications for TeV leptogenesis}

$\mathrm{TeV}$ triplets are thermalized by gauge boson mediated annihilations up to $z \gg 1$ (see fig. 22. The generation of the $B-L$ asymmetry in that case proceeds basically above this $z$ once the relic fraction that survives annihilation start decaying. Sphaleron interactions transform this asymmetry into a $B$ asymmetry up to temperatures $T_{\text {dec }}$ at which their reactions are suddenly decoupled by the spontaneous breaking of the $S U(2)$ symmetry 31 . This constraint combined with $Y_{\Delta_{B}} \sim 10^{-10}$ implies the bound $M_{T} \gtrsim 1.6 \mathrm{TeV}[23]$. This is to be compared with the standard resonant leptogenesis framework in which the fermionic singlet can have a mass well below the $\mathrm{TeV}$ scale. The reason is that while in the standard case the efficiency is basically determined by $\widetilde{m}$, in the fermionic triplet scenario there is a dependence on $M_{T}$ that strongly suppress the efficiency when $M_{T} \sim \mathcal{O}(\mathrm{TeV})$.

Adding flavor effects in the analysis does not allow to relax the bound: in sec. 3 we argued that flavor effects can yield a relevant enhancement of the $B-L$ asymmetry (flavored efficiencies) only when gauge interactions decouple before Yukawa reactions, which requires large values of $\widetilde{m}$, as shown in fig. 3 (right panel). In order to discuss how this could take place for $\mathrm{TeV}$ triplets let us fix $M_{T}=10^{3} \mathrm{GeV}$. Since the sphaleron decoupling temperature is determined by the Higgs mass $\left(m_{h}\right)$ according to $T_{\mathrm{dec}} \simeq[80+$ $\left.0.45\left(m_{h} / \mathrm{GeV}\right)\right] \mathrm{GeV}$ (with $m_{h} \in[114,200] \mathrm{GeV}$ ) [23, 31], fixing $m_{h}=120 \mathrm{GeV}$ sphaleron decoupling will take place at $z_{\mathrm{dec}} \sim 7.5$. At this $z$ Yukawa reactions will overcome gauge interactions provided $\widetilde{m} \gtrsim 3.5 \mathrm{keV} 5$, however since no asymmetry could be produced

\footnotetext{
${ }^{5}$ Such values are possible only if the different Yukawa couplings $\lambda$ are highly fine-tunned.
} 


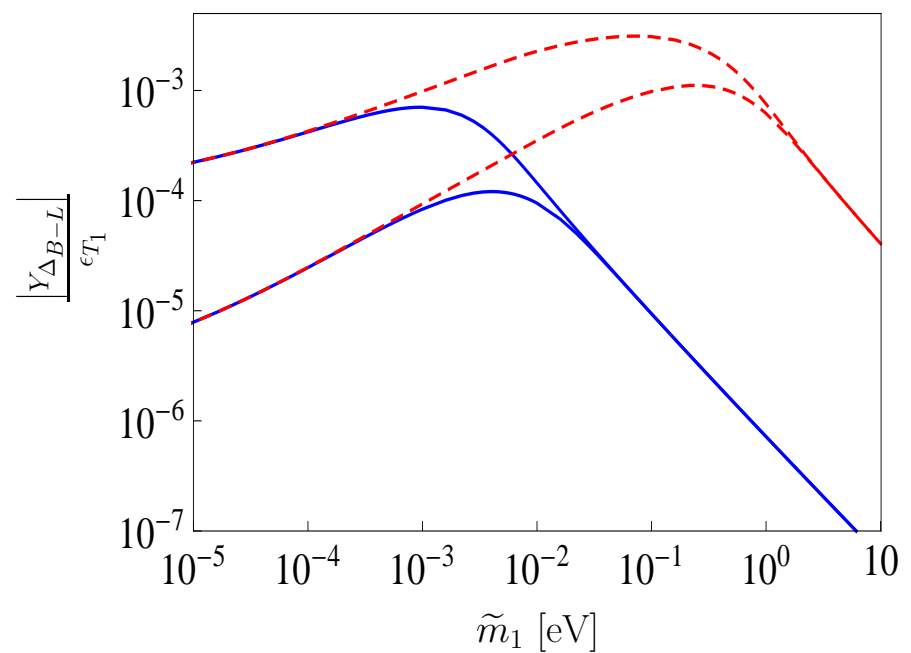

Figure 5: $Y_{\Delta_{B-L}} / \epsilon_{T_{1}}$ as a function of $\widetilde{m}_{1}$ for flavored (dotted red lines) and aligned (solid blue line) cases. The upper (lower) dotted and solid curves correspond to $M_{T_{1}}=10^{12}$ $\mathrm{GeV}\left(M_{T_{1}}=10^{10} \mathrm{GeV}\right)$. The flavored $\mathrm{CP}$ asymmetries and flavor projectors were fixed as in fig. 4 .

until inverse Yukawa decays decouple flavor effects are irrelevant, as this will take place much more above $z_{\mathrm{dec}}$.

Given that the LHC reach for fermion triplets discovery is around 700-800 GeV [32, 34], from the previous discussion, one could be tempted to conclude that any observation of fermionic triplets at the LHC would rule out leptogenesis within this framework. This however is not entirely correct as the asymmetry might be generated by degrees of freedom at higher scales $-T_{2}$ in triplet scenario for example 6 . In this case, since $T_{1}$ will not play any role in the generation of the $B-L$ asymmetry, the lower limit on $M_{T_{1}}$ will no longer hold. However, although $T_{1}$ does not participate in leptogenesis, the asymmetry might still be erased by $T_{1}$ flavor dynamics. The particular flavor structures required to prevent too large $T_{1}$ related washouts may yield particular collider signals that can be used to determine whether leptogenesis might be relevant for the generation of the cosmic baryon asymmetry. In the following we will discuss such a possibility.

Without the loss of generality, we assume the asymmetry is produced by heavy fermion triplet $\left(T_{2}\right)$ dynamics at scales $10^{9} \mathrm{GeV} \lesssim M_{T_{2}} \lesssim 10^{12} \mathrm{GeV}$. Requiring $M_{T_{1}} \lesssim 1 \mathrm{TeV}$ the flavored CP violating asymmetries $\epsilon_{T_{1}}^{\ell_{j}}$ are negligible (see eq. 111) and accordingly $T_{1}$ decays do not generate any asymmetry. Thus, given this triplet mass spectrum, the asymmetry is generated by $T_{2}$ decays (at $z_{2}=M_{2} / T \sim 1$ ) in the two-flavored regime $\left(\ell_{\tau}, \ell_{2}\right)$, where $\ell_{2}$ is an admixture of muon and electron flavors. The calculation of the $B-L$ asymmetry at this stage goes along the same lines as in the case we discussed at the beginning of this section, but taking into account that in this case we deal with $T_{2}$ dynamics. At $z \ll z_{2}$, once muon Yukawa interactions enter in thermodynamical equilibrium, the $Y_{\Delta_{\tau}}$ produced at $z \sim z_{2}$ remains frozen whereas $Y_{\Delta_{2}}$ splits into electron

\footnotetext{
${ }^{6}$ This possibility has been studied in the standard case in both, the one flavor approximation [13] and flavored [14, 15] cases.
} 
and muon asymmetries. The splitting, in the case of $Y_{T_{2}}^{\mathrm{in}}=0$, is determined by [15]

$$
Y_{\Delta_{k}}=\frac{K_{k 2}}{\widetilde{K}_{22}} Y_{\Delta_{2}}
$$

with $k=e, \mu$ and $\sum_{k=e, \mu} K_{k 2} / \widetilde{K}_{22}=1$.

As the temperature drops and reaches $z_{1}=M_{T_{1}} / T \sim 1, T_{1}$ related washout effects become effective and are determined by

$$
\frac{d Y_{\Delta_{i}}\left(z_{1}\right)}{d z_{1}}=-\frac{K_{i 1}}{2 s H z_{1} Y_{\ell}^{\mathrm{Eq}}} \sum_{j=e, \mu, \tau} C_{i j}^{\ell} Y_{\Delta_{j}}\left(z_{1}\right) \gamma_{D_{1}}=-\frac{\kappa_{i 1}}{4} \sum_{j=e, \mu, \tau} C_{i j}^{\ell} Y_{\Delta_{j}}\left(z_{1}\right) K_{1}\left(z_{1}\right) z_{1}^{3},
$$

where $K_{1}\left(z_{1}\right)$ is the modified Bessel function of first-type, the parameter $\kappa_{i 1}=\widetilde{m}_{i 1} / m_{\star}$, with $m_{\star}=\left.8 \pi v^{2} H\right|_{z_{1}=1} / M_{T_{1}}^{2}=2.25 \times 10^{-3} \mathrm{eV}$, and the flavor coupling matrix at this stage is given by [11]

$$
C^{\ell}=\frac{1}{711}\left(\begin{array}{ccc}
221 & -16 & -16 \\
-16 & 221 & -16 \\
-16 & -16 & 221
\end{array}\right) .
$$

A rough estimate of washout effects can be done by simply neglecting flavor coupling i.e. setting $C^{\ell}=\mathbf{I}$ and assuming the $T_{1}$ related washout processes are efficient before sphalerons decouple below the electroweak phase transition. In that case eq. (23) can be analytically integrated and the final baryon asymmetry can be written in terms of flavored asymmetries $\left(Y_{\Delta_{i}}^{\text {in }}\right)$ generated at a high scale (in $T_{2}$ decays) and $T_{1}$ washout related damping factors

$$
Y_{\Delta_{B}} \simeq \sum_{i=e, \mu, \tau} Y_{\Delta_{i}}^{\mathrm{in}} e^{-3 \pi \kappa_{i 1} / 8}
$$

Whether the correct amount of baryon asymmetry can be generated therefore depends not only on $Y_{\Delta_{i}}^{\text {in }}$ but also on the $T_{1}$ related washout parameters $\kappa_{i 1}$. In particular, for any value of $Y_{\Delta_{i}}^{\text {in }}$, there exist $\kappa_{i 1}>\kappa_{i 1}^{\max }$, for which that particular flavored asymmetry will be completely washed out by $T_{1}$ dynamics before sphaleron decoupling and will thus not contribute to $Y_{\Delta_{B}}$. Including flavor dynamics and finite sphaleron decoupling temperature, the values of $\kappa_{i 1}^{\max }$ must be determined numerically, by solving eqs. 23) for a given set of $Y_{\Delta_{i}}^{\text {in }}$ and $M_{T_{1}}$. Generically however, taking into account that $T_{1}$ related washouts are relevant only if $\kappa_{i 1} \gtrsim 1$ we can already distinguish between three cases:

1. $\kappa_{i 1} \ll 1$ for all flavors. In that case all $T_{1}$ washout processes are weak and the baryon asymmetry is determined by high scale dynamics (of $T_{2}$ in our scenario). Successful leptogenesis is possible in principle depending on the (practically unmeasurable) high-scale ( $T_{2}$ related) parameters.

2. $\kappa_{i 1} \ll 1$ and $\kappa_{j 1} \gtrsim 1$. The asymmetry in flavor(s) $j$ will now be subject to possibly strong washouts due to $T_{1}$. However, the asymmetry in flavor(s) $i$ will again be completely determined at the high scale (by $T_{2}$ decays). In general therefore any constraints on $\kappa_{j 1}$ rely on the assumptions about the size of $Y_{\Delta_{i}}^{\text {in }}$. Even if some of $Y_{\Delta_{j}}^{\text {in }}$ would be strongly damped, leptogenesis might (or not) account for the baryon asymmetry. 


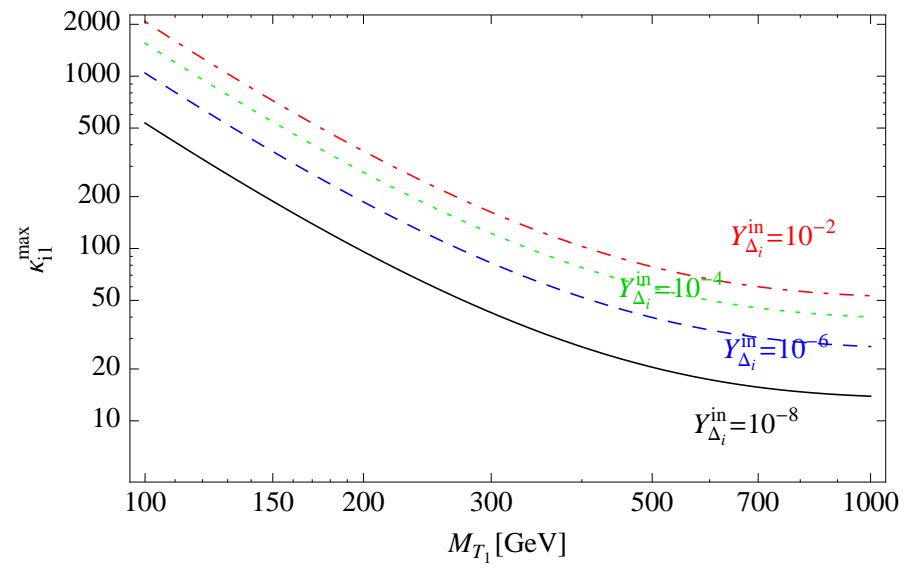

Figure 6: Solutions of $\kappa_{i 1}^{\max }$ for given values of high-scale generated lepton asymmetries $Y_{\Delta_{i}}^{\text {in }}$. See text for details.

3. $\boldsymbol{\kappa}_{\boldsymbol{i} \mathbf{1}} \gtrsim \mathbf{1}$ for all flavors. Washout effects are relevant in all the flavors and the relevant washout parameters can in principle be constrained, depending on the size of $Y_{\Delta_{i}}^{\text {in }}$. Once for a given flavor $\kappa_{i 1}$ reaches $\kappa_{i 1}^{\max }$ the asymmetry in such a flavor will not contribute to $Y_{\Delta_{B}}$ implying that the final baryon asymmetry can be given by

(a) Single flavor: $\kappa_{i 1}<\kappa_{i 1}^{\max }$ and $\kappa_{(j, k) 1}>\kappa_{(j, k) 1}^{\max }$

(b) Two flavors: $\kappa_{(i, j) 1}<\kappa_{(i, j) 1}^{\max }$ and $\kappa_{k 1}>\kappa_{k 1}^{\max }$

(c) Three flavors: $\kappa_{(e, \mu, \tau) 1}<\kappa_{(e, \mu, \tau) 1}^{\max }$

Finally, in order to determine $\kappa_{i 1}^{\max }$ we solve numerically eqs. (23) for given individual $Y_{\Delta_{i}}^{\text {in }}$ ranging from zero up to $10^{-2}$ (the largest values are motivated by completeness in order to cover possible extreme cases such as resonant leptogenesis and possibly large flavor enhancements) and require the final asymmetry $Y_{\Delta_{B}}\left(T_{\text {dec. }}\right)$ to satisfy the experimental constraint $Y_{\Delta_{B}}=[8.52,8.98] \times 10^{-11}$. We plot the obtained solutions (which are independent of lepton flavor, as can be inferred directly from the lepton flavor permutation symmetry of eq. (23)) as contours in the plane of $M_{T_{1}}$ and $\kappa_{i 1}^{\max }$ in figure 6 . We see that successful high scale thermal leptogenesis $\left(Y_{\Delta_{i}}^{\text {in }} \lesssim 10^{-8}\right)$ requires $\kappa_{i 1}<10(500)$ for the $T_{1}$ masses of $M_{T_{1}}=1000(100) \mathrm{GeV}$. On the other hand, in the extreme case $Y_{\Delta_{i}}^{\text {in }} \sim 10^{-2}, T_{1}$ washout parameters as large as $\kappa_{1 i} \lesssim 50(2000)$ are allowed for $T_{1}$ masses of $M_{T_{1}}=1000(100) \mathrm{GeV}$ respectively.

\subsection{Implications for type III see-saw models at Colliders}

Fermionic triplets responsible for neutrino mass generation could be produced directly at colliders and thus shed light on the viability of high scale leptogenesis in type III seesaw scenarios. Existing studies of triplet production at the LHC [32, 33, 34] discuss the signals and backgrounds in detail. The main conclusion is that a fermionic triplet with a mass up to $700 \mathrm{GeV}$ would be accessible at the LHC running at $\sqrt{s}=14 \mathrm{TeV}$ with an integrated luminosity of $100 \mathrm{fb}^{-1}$. The 'smoking gun' signal would be the lepton number 


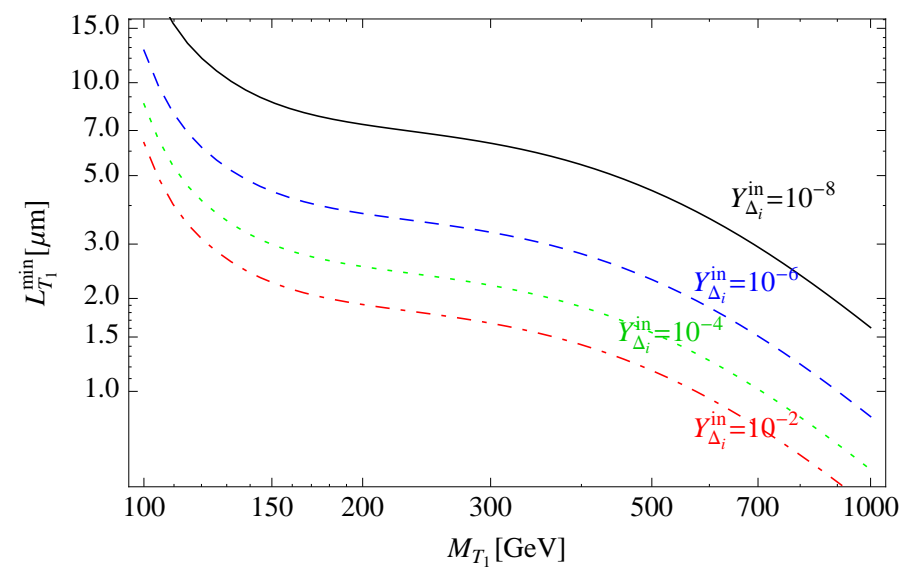

Figure 7: Minimum total $T_{1}$ decay length versus the $T_{1}$ mass for given values of high-scale generated lepton asymmetries $Y_{\Delta_{i}}^{\text {in }}$. See text for details.

violating channel of two same sign leptons and four jets without missing energy. The main SM background comes from $t \bar{t}$ plus jet events which can be sufficiently suppressed by appropriate cuts on $p_{T}$ and missing energy [32, 34].

The flavored decay rates of the triplet are proportional to its Yukawa couplings to light leptons. Therefore, small Yukawa values imply a long lived particle, resulting in displaced secondary vertices in collider detectors. In type III scenarios, the proper decay length of the lightest triplet can range up to $\sim 10 \mathrm{~mm}$ in the minimal setups [34] and more than a meter if three triplets are introduced alltogether [32].

Obviously in the limit of vanishing Yukawa couplings, also the associated washout effects vanish as seen in eq. (25). In order to quantify the threshold, where the washout becomes relevant we use eq. (6) for the triplet partial decay width to a given lepton flavor. The expression is written in an $S U(2)$ invariant way and thus valid in the heavy triplet limit $\left(M_{T_{1}} \gg v\right)$. Close to the electroweak symmetry breaking scale, one needs to work in the mass eigenbasis and take into account kinematic corrections due to finite $W, Z$ and Higgs masses resulting in 38 ]

$$
\begin{aligned}
& \Gamma\left(\ell_{i}\right) \simeq 10^{-12} \kappa_{i}\left(\frac{M_{T_{1}}}{1 \mathrm{TeV}}\right)^{2} \frac{1}{4}\left[2 f_{1}\left(\frac{m_{W}}{M_{T_{1}}}\right)+f_{1}\left(\frac{m_{Z}}{M_{T_{1}}}\right)+f_{0}\left(\frac{m_{h}}{M_{T_{1}}}\right)\right] \mathrm{GeV}, \\
& f_{n}(x)=\left(1-x^{2}\right)^{2}\left(1+2 n x^{2}\right) \Theta(1-x) .
\end{aligned}
$$

Inserting $\kappa_{i 1}^{\max }$ derived in the previous section into the above triplet decay rate (and summing over lepton flavors) gives a lower bound on the proper lifetime of a democratically decaying $T_{1}\left(\kappa_{e} \simeq \kappa_{\mu} \simeq \kappa_{\tau}\right)$ as a function of $M_{T_{1}}$ which is shown in figure 7 . We observe that kinematic effects are important for triplet masses below $150 \mathrm{GeV}$ where also our estimates for the washout cease to be reliable.

Although the experimental resolution of the secondary vertex in the LHC experiments strongly depends on the flavor composition of the decay, it should allow to reconstruct a secondary vertex due to a triplet to no better than around $100 \mu \mathrm{m}$ in the direction orthogonal to the beam axis [32]. Therefore, seeing a triplet induced displaced vertex at 


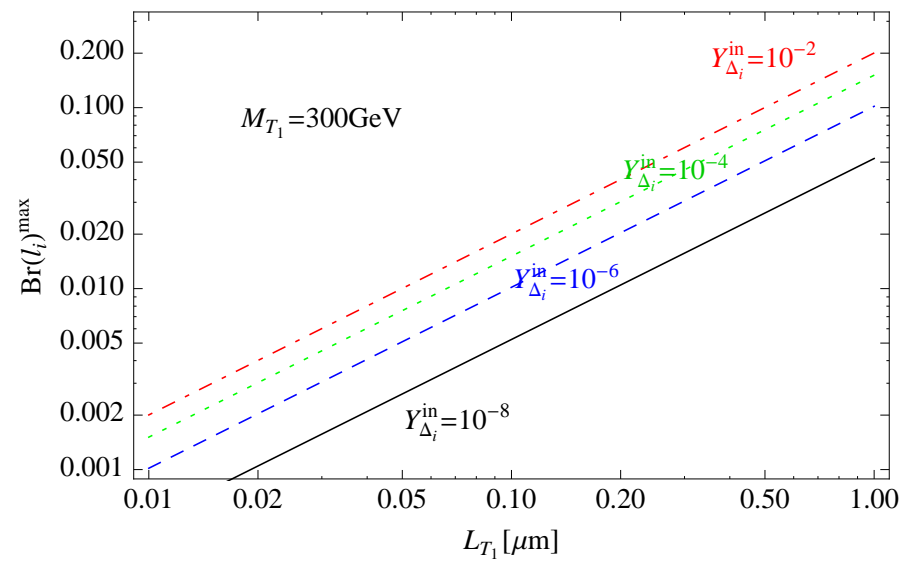

Figure 8: Parameter space regions of $T_{1}$, allowing for successful high scale leptogenesis yielding the given asymmetry values in lepton flavor $i$. Regions below the curves are allowed for each $Y_{\Delta_{i}}^{\text {in }}$ value. $\operatorname{Br}\left(\ell_{i}\right)^{\text {max }}$ stands for the maximum allowed branching ratio of $T_{1}$ to a lepton of flavor $i$. See text for details.

the LHC certainly implies cases 1 or 3(c) of the previous section - a long lived triplet accessible at the LHC is consistent with high scale leptogenesis accounting for the cosmic baryon asymmetry.

On the other hand, completely excluding a pre-existing leptonic asymmetry at the LHC seems quite challenging, even for $Y_{\Delta_{i}}^{\text {in }}=10^{-8}$ as allowed by high scale thermal non-resonant leptogenesis. Cases 2 , $3 \mathrm{a}$ and $3 \mathrm{~b}$ are all characterized by a relatively short lived triplet with its proper length below $10 \mu \mathrm{m}$ for $M_{T_{1}}>150 \mathrm{GeV}$. The maximum proper length excluding the survival of any lepton asymmetries for a given triplet mass is reached for approximately equal decay branching ratios into all lepton flavors. The required improvement in the secondary vertex resolution for this case might be offered by the proposed ILC experiment [35]. Triplets of a couple hundred $\mathrm{GeV}$ may be produced copiously at the ILC with a total cross section $\sigma \sim \mathcal{O}(100 \mathrm{fb})$ [34]. If the proposed detector requirements [35] were realized, the vertexing resolution would be roughly $1 / 3$ of the LHC's detectors which is close to the interesting region discussed above ${ }^{7}[35$.

Finally, if one or more of the flavored branching ratios is suppressed, a lepton asymmetry may survive in that particular channel. In this case, the $T_{1}$ lifetime may be even smaller than indicated in fig. 7, beyond any realistic detector resolution. The condition of surviving lepton asymmetry can then be phrased in terms of an upper bound on the smallest flavored branching ratio, depending on the $T_{1}$ mass and total width. Experimentally the least favorable case is in the transition region between the two regimes, where the proper decay length of the triplet is below the experimental resolution while the hierarchy between the different flavored branching ratios is not very pronounced, making definite statements about the viability of high scale leptogenesis difficult. This situation is visualized in fig. 8 for representative values of $Y_{\Delta_{i}}^{\text {in }}$ and for $M_{T_{1}}=300 \mathrm{GeV}$. Note also that

\footnotetext{
${ }^{7}$ Note that in the detector reference frame, decay lengths of particles of a couple of hundred GeV may receive sizable boost factors.
} 
in order to discriminate between the cases $3 \mathrm{a}$ and $3 \mathrm{~b}$, and the parameter region where high scale leptogenesis is ruled out, all three leptonic decay channels of $T_{1}$ would need to be measured or at least experimentally constrained.

\subsection{Implications for lepton flavor violation in minimal type III $(+\mathbf{I})$ see-saw models}

Recently, it has been shown, that a mixed type I+III see-saw model with a single fermionic singlet and triplet below the $\mathrm{TeV}$ scale naturally arises in a particular grand unified scenario [37, 38]. It belongs to a class of so-called 'minimal' type I and III see-saw scenarios, where the light neutrino mass matrix due to the mixing with only two heavy Majorana states is of rank 2 and the lightest neutrino remains (almost) massless. A particularity of such models is that the neutrino mass scale is fixed and that all the interactions of the heavy mediators are fixed in terms of the measured neutrino oscillation data, a single physical Majorana phase and a complex parameter $z$ [40], which is the single complex rotation angle in the $2 \times 2$ orthogonal matrix $R$ introduced in eq. (5) (we employ conventions as defined in [41]). The presence of $\mathrm{TeV}$ scale triplets can induce lepton flavor violating (LFV) processes which, in the minimal models, are all correlated and their magnitude scales exponentially with $\operatorname{Im} z$. The greatest sensitivity is exhibited in the $\mu-e$ sector by the nuclear conversion experiments, yielding present bounds of the order $\operatorname{Im} z<7$ for the lightest triplet mass of $M_{T}=100 \mathrm{GeV}$ [41]. The proposed next generation PRISM/PRIME experiments [42] are expected to improve the sensitivity down to $\operatorname{Im} z<4$ for the same triplet mass.

From the discussion in the previous sections it is clear, that minimal type III models at the $\mathrm{TeV}$ scale cannot mediate successful leptogenesi. ${ }^{8}$. However, the requirements that the flavor dynamics of the $\mathrm{TeV}$ scale triplets do not wash out any lepton number asymmetries generated at a higher scale by other mechanisms can in these models be phrased entirely in terms of $M_{T}$ and $z$. As such they can then be correlated with the projected sensitivity of future LFV experiments. In particular for the minimal I+III see-saw scenario we plot the constraints imposed by allowing for a high scale lepton asymmetry to survive triplet related washouts for a given $Y_{\Delta}^{\text {in }} \equiv \sum_{i} Y_{\Delta_{i}}^{\text {in }}$ on the parameters of the model $(\operatorname{Im} z$ and $M_{T}$ ) and superimpose them onto the projected sensitivity of future $\mu-e$ conversion experiments. In this procedure we marginalize over unknown phases, individual $Y_{\Delta_{i}}^{\text {in }}$ (while keeping their sum fixed) and also Re $z$. The results are shown in figure 9 for normal (on the left side) and inverted (on the right side) neutrino mass hierarchy. The results for the minimal pure type III models are similar. We observe that in the minimal models, requiring a high-scale lepton asymmetry to survive triplet related washouts rules out LFV effects which could be detected in the foreseeable future. Conversely, observation of

\footnotetext{
${ }^{8}$ It has been proposed that resonant leptogenesis could be viable in the $\mathrm{TeV}$ scale minimal $\mathrm{I}+\mathrm{III}$ model 38. At first glance, it seems like an interesting possibility, since the fermionic singlet mediator would not suffer from the gauge reaction related strong washouts plaguing $\mathrm{TeV}$ triplet leptogenesis. However, at temperatures above the electroweak phase transition the mixing between $S U(2)$ singlets and triplets is highly suppressed and a large resonant enhancement due to the interference with the wavefunction diagram in figure 1 cannot occur [39].
} 

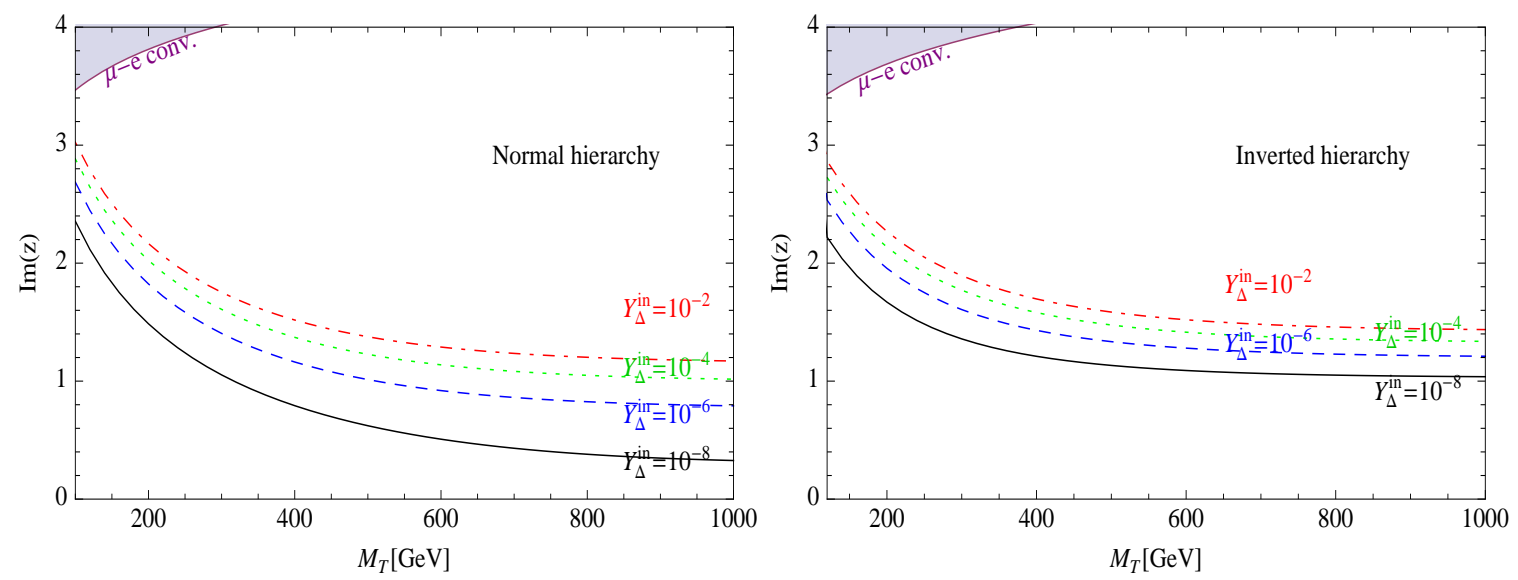

Figure 9: Correlation of leptogenesis bounds (area below the contours is allowed for a given $Y_{\Delta}^{\text {in }}$ ) and projected $\mu-e$ conversion sensitivity of PRISM/PRIME [42] (shaded area) on the parameters of the minimal I+III model $\left(\operatorname{Im} z\right.$ and $\left.M_{T}\right)$. See text for details.

minimal see-saw models at the LHC and detection of the associated LFV signals in the next generation of $\mu-e$ nuclear conversion experiments would disfavor leptogenesis as the mechanism for the generation of the cosmic baryon asymmetry.

Finally, within minimal scenarios, LHC (and ILC) may yield additional information on the size of the Yukawa couplings. Since in order to have an effective washout, $\operatorname{Im} z \gtrsim 1$ is required as seen on fig. 9 for both normal and inverted hierarchies, predictions for correlations between different flavored branching ratios emerge, as discussed at length in [34]. For example, in the case of a small $\theta_{13}$ [38] the inverted hierachy gives a clear prediction

$$
\frac{\operatorname{Br}(\tau)}{\operatorname{Br}(\mu)}=\tan ^{2} \theta_{23}
$$

and measuring the electron channel may disprove a large value of $\operatorname{Im} z$. A similar statement, although less elegant, can be made in the case of normal hierarchy, where $\operatorname{Br}(e)$ and the total decay width is a function of $z$ only.

\section{Conclusions}

The generation of a lepton asymmetry, via the decays of fermionic $S U(2)$ triplets, can take place in two possible regimes: a "gauge regime" in which gauge reactions decouple at lower temperatures than Yukawa induced inverse decays. In that case the relic fraction of triplets that survives the gauge boson mediated annihilation processes will decay producing an amount of $B-L$ asymmetry whose value will strongly depend on $M_{T}$. Conversely, in the "Yukawa regime", at low temperatures, the triplet distribution is driven to thermal equilibrium by Yukawa inverse decay processes, even after gauge interactions have switched off. In contrast to the "gauge regime", in this case the amount of $B-L$ generated in $T$ decays is not sensitive to $M_{T}$ and is fixed by $\widetilde{m}$. We have determined the $\widetilde{m}-M_{T}$ regions that define both regimes. After identifying these regions we have 
studied the effects of light lepton flavors on the generation of the $B-L$ asymmetry. We have shown that in the "gauge regime" the effects have a minor influence as they barely reach fractions of a percent. Instead, in the "Yukawa regime", as in the standard case, the enhancement of the final $B-L$ can be large, readily reaching the order of magnitude level.

We have shown that the requirement of successful $T_{1}$ leptogenesis constrains the dynamics of $\mathrm{TeV}$ triplets to lie in the "gauge regime", and therefore the inclusion of light lepton flavor effects in the calculation of the $B-L$ asymmetry has basically no significant effect. This implies that even after including flavor the bound $M_{T_{1}} \gtrsim 1.6 \mathrm{TeV}$, found in the one flavor approximation [23], still holds. Since fermionic triplets will be produced at the LHC if their masses are below $\sim 1 \mathrm{TeV}[32$ their observation will automatically preclude them from explaining the Cosmic baryon asymmetry via $T_{1}$ leptogenesis. However, it will not necessarily exclude the case for high scale leptogenesis, due to $T_{2}$ or other mechanisms. In fact, as we have argued, the $B-L$ asymmetry can be built up at a very early age $\left(T \ggg M_{T_{1}}\right)$ and can possibly survive $T_{1}$ washout processes. We have shown that $T_{1}$ collider observables could, in principle, be used either to strengthen or rule out the case for high scale leptogenesi ${ }^{9}$ (there have been recent related attempts to invalidate leptogenesis [43] or high scale baryogenesis [44] using collider observables). Finally, we have shown how in minimal see-saw models, LFV observables can also be employed in order to over-constrain the region of parameters of $\mathrm{TeV}$ scale Majorana electroweak triplets allowing for successful high scale leptogenesis.

\section{Acknowledgements}

We acknowledge many enlightening conversions on the subject with Enrico Nardi, Juan Racker and Ilja Doršner. We are especially thankful to Enrico Nardi for his suggestions and comments on the manuscript. D.A.S also wants to thank the theoretical physics group at the Josef Stefan Institute for their hospitality during the completion of this work and is supported by a Belgian FNRS postdoctoral fellowship. This work is supported in part by the Slovenian Research Agency, by the European Commission RTN network, Contract No. MRTN-CT-2006-035482 (FLAVIAnet) and by the Deutsche Forschungsgemeinschaft via the Junior Research Group "SUSY Phenomenology" within the Collaborative Research Centre 676 "Particles, Strings and the Early Universe".

\section{A Conventions and notation}

In this appendix we fix our conventions and present the relevant equations used in the calculations discussed in sections 2 and 3 . Some of the formulae are quite standard, however, for completeness, we include them. As has been mentioned we used Maxwell-

\footnotetext{
${ }^{9}$ Note that a similar argument could apply for other mechanisms of high scale baryogenesis, since sphaleron processes will tend to equilibrate any $B$ asymmetry with $L$, which could in term be washed out by light Majorana triplet interactions.
} 
Boltzmann distributions for the equilibrium number densities, namely

$$
n_{T}^{\mathrm{eq}}(z)=\frac{M_{T}^{3}}{\pi^{2}} \frac{K_{2}(z)}{z}, \quad n_{\ell}^{\mathrm{eq}}(z)=\frac{2 M_{T}^{3}}{\pi^{2} z^{3}},
$$

where $z=M_{T} / T$ and $K_{2}(z)$ is the modified Bessel function of the second-type. With this approximation the energy density $\rho(z)$ and pressure $p(z)$ becomes

$$
\rho(z)=\frac{3 M_{T}^{4}}{z^{4} \pi^{2}} g_{*}, \quad p(z)=\frac{M_{T}^{4}}{z^{4} \pi^{2}} g_{*},
$$

where $g_{*}=\sum_{i=\text { All species }} g_{i}$ is the number of standard model relativistic degrees of freedom (118 for $T \gg 300 \mathrm{GeV}$ ). Accordingly, the expansion rate of the Universe and entropy density can be written as

$$
H(z)=\sqrt{\frac{8 g_{*}}{\pi}} \frac{M_{T}^{2}}{M_{\text {Planck }}} \frac{1}{z^{2}}, \quad s(z)=\frac{4 M_{T}^{3}}{z^{3} \pi^{2}} g_{*} .
$$

The decay and annihilation reaction densities in eqs. (14) and (15) are given by

$$
\begin{aligned}
\gamma_{D_{\alpha}}(z) & =\frac{1}{8 \pi^{3}} \frac{M_{T_{\alpha}}^{5}}{v^{2}} \frac{K_{1}(z)}{z} \widetilde{m}_{\alpha}, \\
\gamma_{A_{\alpha}}(z) & =\frac{M_{T_{\alpha}}^{4}}{64 \pi^{4}} \int_{4}^{\infty} d x \frac{\sqrt{x} K_{1}(z \sqrt{x}) \widehat{\sigma}_{A}(x)}{z},
\end{aligned}
$$

where $K_{1}(z)$ is the modified Bessel function of first-type and $x=s / M_{T}^{2}$. The reduced cross section in (33) accounts for the gauge boson mediated s-channel processes $T_{\alpha} T_{\alpha} \leftrightarrow \ell \bar{\ell}$ and $T_{\alpha} T_{\alpha} \leftrightarrow q \bar{q}$ and the $\mathrm{t}$ and $\mathrm{u}$-channel triplet mediated process $T_{\alpha} T_{\alpha} \leftrightarrow A_{\mu} A^{\mu}\left(A^{\mu}=\right.$ $\left.W_{A}^{\mu}, B^{\mu}\right)$. For $\widehat{\sigma}_{A}(x)$ we used the expression given in ref. [21]:

$$
\widehat{\sigma}_{A}(x)=\frac{6 g^{4}}{\pi}\left(1+\frac{2}{x}\right) r+\frac{2 g^{4}}{\pi}\left[3\left(1+\frac{4}{x}-\frac{4}{x^{2}}\right) \log \left(\frac{1+r}{1-r}\right)-\left(4+\frac{17}{x}\right) r\right],
$$

with $r=\sqrt{1-4 / x}$.

Neglecting flavor coupling, i.e $C^{\ell}=I$ eq. 15 can be analytically integrated (by using its integrating factor). Assuming a vanishing initial asymmetry $\left(Y_{\Delta_{i}}^{\text {in }}\left(z_{0}\right)=0\right)$ the result reads

$$
Y_{\Delta_{i}}(z)=-\epsilon_{T_{\alpha}}^{\ell_{i}} Y_{T_{\alpha}}^{\mathrm{Eq}}\left(z_{0}\right) \eta_{i \alpha}(z)
$$

The flavored efficiency function $\eta_{i \alpha}(z)$ determines the evolution of the $\Delta_{i}$ asymmetry at any $z$. Using the approximation $Y_{T_{\alpha}}(z)+Y_{T_{\alpha}}^{\mathrm{Eq}}(z) \simeq 2 Y_{T_{\alpha}}^{\mathrm{Eq}}(z)$ it can be written as

$$
\eta_{i \alpha}(z)=\frac{1}{Y_{T_{\alpha}}^{\mathrm{Eq}}\left(z_{0}\right)} \int_{z_{0}}^{z} d z^{\prime} \frac{\gamma_{D_{\alpha}}}{\gamma_{D_{\alpha}}+2 \gamma_{A_{\alpha}}} \frac{d Y_{T_{\alpha}}\left(z^{\prime}\right)}{d z^{\prime}} e^{-\sum_{\alpha} \int_{z^{\prime}}^{z} d z^{\prime \prime} P_{i \alpha}\left(z^{\prime \prime}\right)}
$$

where freeze-out of $\Delta_{i}$ takes place at $z_{f}\left(z_{0} \ll z_{f}\right)$ and

$$
P_{i \alpha}(z)=\frac{K_{i \alpha}}{2 Y_{\ell}^{\mathrm{Eq}}(z)} \frac{\gamma_{D_{\alpha}}(z)}{s(z) H(z) z} \text {. }
$$


For temperatures $T \gtrsim 10^{13} \mathrm{GeV}$, for which the flavor composition of the lepton doublet states is irrelevant, $K_{i \alpha}=1$.

The final $B-L$ asymmetry is determined according to

$$
Y_{\Delta_{B-L}}=\sum_{i=e, \mu, \tau} Y_{\Delta_{i}}=3 \times \sum_{i=e, \mu \tau} \epsilon_{T_{\alpha}}^{\ell_{i}} Y_{T_{\alpha}}^{\mathrm{Eq}}\left(z_{0}\right) \eta_{i \alpha}\left(z_{f}\right)
$$

where $\eta_{i \alpha}\left(z_{f}\right)$ defines the efficiency factor for flavor $i$ and the factor 3 comes from the triplet degrees of freedom.

\section{References}

[1] G. Hinshaw et al. [WMAP Collaboration], Astrophys. J. Suppl. 180, 225 (2009) arXiv:0803.0732 [astro-ph]].

[2] A. D. Sakharov, Pisma Zh. Eksp. Teor. Fiz. 5 (1967) 32 [JETP Lett. 5 (1967 SOPUA,34,392-393.1991 UFNAA,161,61-64.1991) 24].

[3] A. D. Dolgov, Phys. Rept. 222, 309 (1992).

[4] V. A. Kuzmin, V. A. Rubakov and M. E. Shaposhnikov, Phys. Lett. B 155, 36 (1985).

[5] P. Minkowski, Phys. Lett. B 67421 (1977); T. Yanagida, in Proc. of Workshop on Unified Theory and Baryon number in the Universe, eds. O. Sawada and A. Sugamoto, KEK, Tsukuba, (1979) p.95; M. Gell-Mann, P. Ramond and R. Slansky, in Supergravity, eds P. van Niewenhuizen and D. Z. Freedman (North Holland, Amsterdam 1980) p.315; P. Ramond, Sanibel talk, retroprinted as hep-ph/9809459; S. L. Glashow, in Quarks and Leptons, Cargèse lectures, eds M. Lévy, (Plenum, 1980, New York) p. 707; R. N. Mohapatra and G. Senjanović, Phys. Rev. Lett. 44, 912 (1980).

[6] M. Fukugita and T. Yanagida, Phys. Lett. B 174, 45 (1986).

[7] For a review on standard leptogenesis see: S. Davidson, E. Nardi and Y. Nir, Phys. Rept. 466, 105 (2008) arXiv:0802.2962 [hep-ph]].

[8] R. Barbieri, P. Creminelli, A. Strumia and N. Tetradis, Nucl. Phys. B 575, 61 (2000) (for the updated version of this paper see arXiv:hep-ph/9911315]).

[9] G. F. Giudice, A. Notari, M. Raidal, A. Riotto and A. Strumia, Nucl. Phys. B 685, 89 (2004) arXiv:hep-ph/0310123.

[10] A. Abada, S. Davidson, A. Ibarra, F. X. Josse-Michaux, M. Losada and A. Riotto, JHEP 0609 (2006) 010 arXiv:hep-ph/0605281.

[11] E. Nardi, Y. Nir, E. Roulet and J. Racker, JHEP 0601, 164 (2006) arXiv:hepph/0601084; A. Abada, S. Davidson, F. X. Josse-Michaux, M. Losada and A. Riotto, JCAP 0604 (2006) 004 arXiv:hep-ph/0601083. 
[12] E. Nardi, Y. Nir, J. Racker and E. Roulet, JHEP 0601, 068 (2006) arXiv:hep$\mathrm{ph} / 0512052$.

[13] P. Di Bari, Nucl. Phys. B 727, 318 (2005) arXiv:hep-ph/0502082.

[14] G. Engelhard, Y. Grossman, E. Nardi and Y. Nir, Phys. Rev. Lett. 99, 081802 (2007) arXiv:hep-ph/0612187]; O. Vives, Phys. Rev. D 73, 073006 (2006) [arXiv:hepph/0512160].

[15] S. Antusch, P. Di Bari, D. A. Jones and S. F. King, arXiv:1003.5132 [hep-ph].

[16] J. Schechter and J. W. F. Valle, Phys. Rev. D 22, 2227 (1980); G. Lazarides, Q. Shafi and C. Wetterich, Nucl. Phys. B 181, 287 (1981); R. N. Mohapatra and G. Senjanovic, Phys. Rev. D 23, 165 (1981); C. Wetterich, Nucl. Phys. B 187, 343 (1981);

[17] R. Foot, H. Lew, X. G. He and G. C. Joshi, Z. Phys. C 44, 441 (1989).

[18] T. Hambye and G. Senjanovic, Phys. Lett. B 582, 73 (2004) arXiv:hep-ph/0307237.

[19] S. Antusch and S. F. King, Phys. Lett. B 597, 199 (2004) arXiv:hep-ph/0405093.

[20] S. Antusch, Phys. Rev. D 76, 023512 (2007) arXiv:0704.1591 [hep-ph]].

[21] T. Hambye, Y. Lin, A. Notari, M. Papucci and A. Strumia, Nucl. Phys. B 695, 169 (2004) arXiv:hep-ph/0312203.

[22] W. Fischler and R. Flauger, JHEP 0809 (2008) 020 arXiv:0805.3000 [hep-ph]].

[23] A. Strumia, Nucl. Phys. B 809, 308 (2009) arXiv:0806.1630 [hep-ph]].

[24] A. Pilaftsis and T. E. J. Underwood, Nucl. Phys. B 692, 303 (2004) arXiv:hep$\mathrm{ph} / 0309342$.

[25] A. Pilaftsis and T. E. J. Underwood, Phys. Rev. D 72, 113001 (2005) arXiv:hepph/0506107.

[26] T. Schwetz, M. Tortola and J. W. F. Valle, New J. Phys. 10, 113011 (2008) arXiv:0808.2016 [hep-ph]].

[27] J. A. Casas and A. Ibarra, Nucl. Phys. B 618, 171 (2001) arXiv:hep-ph/0103065.

[28] L. Covi, E. Roulet and F. Vissani, Phys. Lett. B 384, 169 (1996) arXiv:hep$\mathrm{ph} / 9605319$.

[29] E. Nardi, J. Racker and E. Roulet, JHEP 0709, 090 (2007) arXiv:0707.0378 [hep$\mathrm{ph}]$.

[30] J. A. Harvey and M. S. Turner, Phys. Rev. D 42, 3344 (1990).

[31] Y. Burnier, M. Laine and M. Shaposhnikov, JCAP 0602, 007 (2006) arXiv:hep$\mathrm{ph} / 0511246$. 
[32] R. Franceschini, T. Hambye and A. Strumia, Phys. Rev. D 78, 033002 (2008) arXiv:0805.1613 [hep-ph]].

[33] F. del Aguila and J. A. Aguilar-Saavedra, Nucl. Phys. B 813 (2009) 22 arXiv:0808.2468 [hep-ph]].

[34] A. Arhrib, B. Bajc, D. K. Ghosh, T. Han, G. Y. Huang, I. Puljak and G. Senjanovic, arXiv:0904.2390 [hep-ph].

[35] T. Behnke et al. [ILC Collaboration], arXiv:0712.2356 [physics.ins-det].

[36] G. Aad et al. [The ATLAS Collaboration], arXiv:0901.0512 [hep-ex].

[37] B. Bajc and G. Senjanović, JHEP 0708 (2007) 014 [arXiv:hep-ph/0612029].

[38] B. Bajc, M. Nemevšek and G. Senjanović, Phys. Rev. D 76 (2007) 055011 arXiv:hepph/0703080.

[39] S. Blanchet and P. Fileviez Perez, JCAP 0808 (2008) 037 [arXiv:0807.3740 [hep-ph]].

[40] A. Ibarra and G. G. Ross, Phys. Lett. B 591 (2004) 285 [arXiv:hep-ph/0312138].

[41] J. F. Kamenik and M. Nemevšek, JHEP 0911 (2009) 023 arXiv:0908.3451 [hep-ph]].

[42] C. Ankenbrandt et al., arXiv:physics/0611124; http://jparc.jp/NuclPart/pac_0701/pdf/P21-LOI.pdf; http://jparc.jp/NuclPart/pac_0606/pdf/p20-Kuno.pdf.

[43] J. M. Frere, T. Hambye and G. Vertongen, JHEP 0901 (2009) 051 arXiv:0806.0841 [hep-ph]].

[44] S. Blanchet, Z. Chacko and R. N. Mohapatra, Phys. Rev. D 80, 085002 (2009) arXiv:0812.3837 [hep-ph]]. 